\title{
BMJ Open Duration of second victim symptoms in the aftermath of a patient safety incident and association with the level of patient harm: a cross-sectional study in the Netherlands
}

\author{
Kris Vanhaecht, ${ }^{1,2}$ Deborah Seys, ${ }^{1}$ Loes Schouten, ${ }^{3}$ Luk Bruyneel, ${ }^{1,4}$ \\ Ellen Coeckelberghs, ${ }^{1}$ Massimiliano Panella, ${ }^{5}$ Gerda Zeeman, ${ }^{6}$ for the Dutch Peer \\ Support Collaborative Research Group
}

To cite: Vanhaecht K, Seys D, Schouten L, et al. Duration of second victim symptoms in the aftermath of a patient safety incident and association with the level of patient harm: a cross-sectional study in the Netherlands. BMJ Open 2019;9:e029923. doi:10.1136/ bmjopen-2019-029923

- Prepublication history for this paper is available online. To view these files, please visit the journal online (http://dx.doi. org/10.1136/bmjopen-2019029923).

Received 19 February 2019

Revised 28 April 2019

Accepted 21 June 2019

D) Check for updates

(C) Author(s) (or their employer(s)) 2019. Re-use permitted under CC BY-NC. No commercial re-use. See rights and permissions. Published by BMJ.

For numbered affiliations see end of article.

Correspondence to

Dr Deborah Seys;

Deborah.seys@kuleuven.be

\section{ABSTRACT}

Objectives To describe healthcare providers' symptoms evoked by patient safety incidents (PSIs), the duration of these symptoms and the association with the degree of patient harm caused by the incident.

Design Cross-sectional survey.

Setting 32 Dutch hospitals that participate in the 'Peer Support Collaborative'.

Participants 4369 healthcare providers (1619 doctors and 2750 nurses) involved in a PSI at any time during their career. Interventions All doctors and nurses working in direct patient care in the 32 participating hospitals were invited via email to participate in an online survey.

Primary and secondary outcome measures Prevalence of symptoms, symptom duration and its relationship with the degree of patient harm.

Results In total 4369 respondents were involved in a PSI and completely filled in the questionnaire. Of these, 462 reported having been involved in a PSI with permanent harm or death during the last 6 months. This had a personal, professional impact as well as impact on effective teamwork requirements. The impact of a PSI increased when the degree of patient harm was more severe. The most common symptom was hypervigilance (53.0\%). The three most common symptoms related to teamwork were having doubts about knowledge and skill $(27.0 \%)$, feeling unable to provide quality care $(15.6 \%)$ and feeling uncomfortable within the team (15.5\%). PSI with permanent harm or death was related to eightfold higher likelihood of provider-related symptoms lasting for more than 1 month and ninefold lasting longer than 6 months compared with symptoms reported when the PSI caused no harm.

Conclusion The impact of PSI remains an underestimated problem. The higher the degree of harm, the longer the symptoms last. Future studies should evaluate how these data can be integrated in evidence-based support systems.

\section{INTRODUCTION}

It is estimated that patient safety incidents (PSIs) occur in at least one out of seven

\section{Strengths and limitations of this study}

- This study explores an underinvestigated area in the field of patient safety and quality care: duration of healthcare provider second victim symptoms in the aftermath of a patient safety incident and its relationship with the degree of patient harm.

- The very large sample size $(n=6508)$ as well as the large number of included Dutch hospitals $(n=32)$ representing a cross-section of urban/rural, small/ large, (non)-academic and (non)-teaching hospitals.

- In this study, the prevalence of symptoms was based on dichotomous questions (present or absent) and not evaluated by means of validated questionnaires.

- This study is based on data of approximately onethird $(n=32)$ of all Dutch hospitals. The results are not representative for all the healthcare organisations in the Netherlands or anywhere else in the world.

- Response bias is an inherent limitation related to surveys, as also pertains to the study now reported, as well as recall bias since the self-reported presence or absence of symptoms was purely based on the respondents' recollection.

hospitalised patients. ${ }^{1}$ This mostly includes incidents that cause no harm (near misses) but also incidents with temporary harm, permanent harm or death (adverse events). ${ }^{2}$ In addition to the tremendous impact on patients and their family (first victims), the well-being of involved healthcare providers (second victims) may also be significantly affected. ${ }^{3}$ Although there is criticism on the term second victims, no alternative term is available. ${ }^{4}$ Moreover, PSI may harm the reputation of the healthcare department and entire organisation (third victims) and reduce trust in healthcare providers in general. ${ }^{5}$ 
Recent studies indicate that almost $80 \%$ of healthcare providers are involved in a near miss or adverse event at least once in their career and that they were emotionally affected. ${ }^{6}$ The psychological impact depends on individual, situational and organisational aspects and affects both personal well-being and professional functioning. ${ }^{8-10}$ Anxiety, fear, guilt, distress, frustration, anger and feeling insufficient are the most frequently cited symptoms. ${ }^{11}{ }^{12}$ In addition, healthcare providers report a significant workhome interference, greater risk of burn-out and higher intention to leave the job. ${ }^{13}$

More and more hospitals realise that they have a role in providing an institutional support system to meet second victims needs. ${ }^{12}{ }^{14-16}$ Healthcare professionals involved in a PSI may have an increased likelihood developing post-traumatic stress disorder (PTSD) ${ }^{14}$ One of the criteria for PTSD, based on the latest criteria set by the Diagnostic and Statistical Manual of Mental Disorders, Fifth Edition (DSM-5), is that the symptoms last for more than 1 month. ${ }^{15}$ Current literature is lacking in information on duration of symptoms suffered by second victims. This hinders the development of evidence-based institutional support programmes. This study aims to describe the prevalence and duration of healthcare provider self-reported symptoms evoked by PSI, and the association between these symptoms and the degree of patient harm caused by the incident.

\section{METHODS \\ Setting}

This study was conducted at 32 hospitals in the Netherlands that participate in the 'Peer Support Collaborative'. The 32 hospitals represent approximately one-third of all Dutch hospitals and comprise a variety of large and small, rural and urban, (non)-teaching and (non)-academic hospitals. These hospitals are among the first group of Dutch hospitals who expressed the wish to implement an organisational peer support programme for second victims in the aftermath of a PSI, patient complaint or lawsuit. This collaborative aims to determine the needs of their second victims and to define a peer support programme that fits their specific organisational patient safety culture and now reports their first insights. As part of the activities of the collaborative, each hospital distributed a standard questionnaire among their doctors and nurses in order to determine their specific needs.

\section{Participants}

Three rounds of data collection were conducted depending on the year of enrolment of the participating hospital in the peer support collaborative. Ten hospitals conducted the survey between 14 April and 15 June 2016, 9 hospitals between 1 February and 30 March 2017 and 13 hospitals between 2 June 2018 and 23 November 2018. All doctors and nurses working in direct patient care in the 32 participating hospitals were invited via email to participate in an online survey under the auspices of the
KU Leuven. Respondents could only participate once. The online survey was available during 4 weeks. The contact person participating in the peer support collaborative of each hospital distributed the web survey in his or her hospital and one email reminder was sent to all doctors and nurses in the third week of the study period. According to the regulations in Dutch hospitals, in the survey, it was mentioned that filling in the questionnaire implied informed consent to participate. Participation was entirely voluntary, and confidentiality and anonymity were guaranteed.

\section{Measurements}

The questionnaire contained four distinct parts. The first part pertained to respondent demographics (profession, years of experience, years of work experience in this hospital, type of ward (surgical of non-surgical) and gender). The second part surveyed the presence or absence of personal involvement in PSI (during the entire career and during the previous 6 months) and the degree of harm (impact of PSI on the patient) (no harm, temporary harm, permanent harm and death). This part started with more information about what a PSI is and the difference with a not-patient safety related dramatic event. Third, personal involvement and symptoms related to the PSI were measured. These 11 symptoms were selected based on a literature research. ${ }^{9}$ Response categories for the duration of the symptoms were 'none', 'some hours', 'a day', 'a week', 'a month', '2-6 months', '6-12 months' and 'more than 1 year'. The questionnaire was based on previous research ${ }^{13} 17-19$ and redesigned by the participating hospitals during a meeting of the peer support network.

\section{Statistics}

Only completely filled in questionnaires were included in the analysis, except for the prevalence rate. Demographic variables are reported and descriptive data as well as the recollection of the degree of PSI-related patient harm during the entire career and during the previous 6 months. Recollection of the presence or absence of symptoms perceived in the aftermath of a PSI is represented in individual prevalence ratios. This ratio indicates whether the specific symptom lasted for more than 1 month and for more than 6 months, and their prevalence pertaining to PSI with no patient harm, temporary harm, permanent harm and death. We opted to use prevalence ratios as these are less prone to overestimate associations compared with using ORs. ${ }^{16}$ Descriptive analyses and prevalence ratios were produced using SAS V.9.4. This manuscript is compliant to the Strengthening the Reporting of Observational Studies in Epidemiology recommendations.

\section{Patient and public involvement}

There was no patient or public involvement in this study. 
Table 1 Demographic information

Doctors (1619) Nurses (2750)

\begin{tabular}{lll}
\hline Gender & & \\
Female & $898(55.5 \%)$ & $2531(92.0 \%)$ \\
\hline $\begin{array}{l}\text { Years of experience } \\
\text { (mean } \pm \text { SD) }\end{array}$ & $11.2 \pm 8.8$ & $18.1 \pm 12.1$ \\
\hline
\end{tabular}

Type of department

\begin{tabular}{lll} 
Surgical department & $540(35.4 \%)$ & $751(29.1 \%)$ \\
\hline $\begin{array}{l}\text { Non-surgical department } \\
\text { Surgical and non-surgical }\end{array}$ & $73(59.8 \%)$ & $1506(58.3 \%)$ \\
department & $327(12.6 \%)$ \\
\hline
\end{tabular}

\section{RESULTS}

A total of 6508 participants in 32 participating hospitals completed the questionnaire. In total 5572 respondents (1938 doctors and 3634 nurses) reported that they had been involved in a PSI at least once during their career $(85.6 \%)$ and 4369 (1619 doctors and 2750 nurses) choose to completely fill out the questions regarding symptoms and were included in this study. Table 1 summarises the demographic information of the 4369 respondents.

During their entire career, over half of respondents had only been involved in a PSI with no harm whereas approximately $45 \%$ had been involved in a PSI with permanent harm or with death (table 2). During the previous 6 months, $80 \%$ of the respondents had only been involved in a PSI with no harm or temporary harm whereas one in five reported having been involved in a PSI with permanent harm or death. Doctors reported more involvement in PSI resulting in permanent harm or death compared with nurses.

\section{Symptoms}

Figure 1 depicts an overview of the 11 symptoms and their frequency of occurrence. The most frequently reported symptom was hypervigilance which bothered $53.0 \%$ of the respondents for more than 1 month. In frequency of occurrence, this was followed by doubts about knowledge and skill $(27.0 \%)$, stress $(25.8 \%)$, shame $(24.7 \%)$, flashbacks $(23.3 \%)$, fear $(19.0 \%)$, feeling unable to provide quality care $(15.6 \%)$, feeling uncomfortable within team $(15.5 \%)$, avoiding risks $(13.0 \%)$, feeling unhappy and dejected (12.5\%) and difficulty sleeping (10.8\%). The prevalences of symptoms with a duration of more than 6 months were respectively hypervigilance $(23.6 \%)$, flashbacks $(8.7 \%)$, shame $(8.2 \%)$, doubts about knowledge and skill $(8.1 \%)$, stress $(6.8 \%)$, fear $(6.3 \%)$, feeling unable to provide quality care $(4.8 \%)$, avoiding risks $(4.5 \%)$, feeling uncomfortable within team $(4.2 \%)$, difficulty sleeping $(2.8 \%)$ and feeling unhappy and dejected $(2.6 \%)$. Of note, in total, $3.7 \%$ of the responding doctors and $4.0 \%$ of the nurses reported never having had any symptom while a significant portion of these providers were personally involved in PSI resulting in permanent harm or death.

Table 3 depicts an overview of the different symptoms which persisted for more than 1 month for each degree of harm. The impact of a PSI with temporary harm, permanent harm and death are more profound than a PSI with no harm, showing up to two, three, four and eightfold symptom prevalence. While the reported symptoms demonstrate a personal impact (difficulty sleeping, fear, stress, shame etc) organisations need also be aware that some symptoms also have an impact on the teamwork, patient safety and ability to provide quality care. For example, $27 \%$ of the respondents mentioned that they have doubts about knowledge and skill for more than 1 month, $15.6 \%$ feel unable to provide quality care and $15.5 \%$ feel uncomfortable within their team.

Table 4 presents symptoms that lasted more than 6 months following the most severe PSI that was recollected by the respondent. The impact of PSI with temporary harm, permanent harm and death are more profound than a PSI with no harm, showing up to two, three, four, five, six, seven and ninefold prevalence of symptoms. Hypervigilance is the symptom which remained the most constant and independent of the degree of harm.

\section{DISCUSSION}

The impact of PSI on healthcare professionals remains an underestimated problem. This study adds relevant information on the prevalence and (long-lasting) duration of several self-reported symptoms and their relation with the degree of patient harm. Almost half of the respondents reported their involvement in a PSI resulting in permanent harm or death at least once during their entire career, while one in five reported involvement in a PSI resulting in permanent harm or death during the previous 6 months. Those latter 462 healthcare professionals were

Table 2 Prevalence for the different degree of harm (reporting only patient safety incident (PSI) with the highest reported degree of harm)

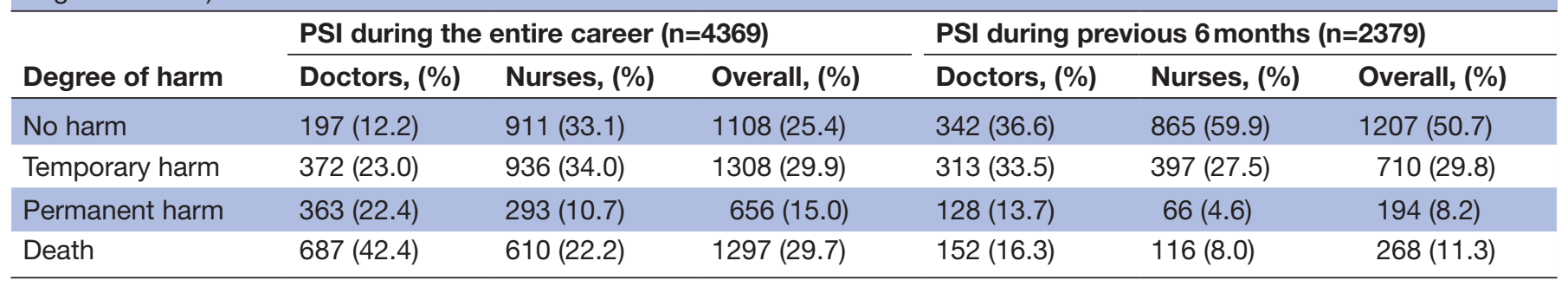




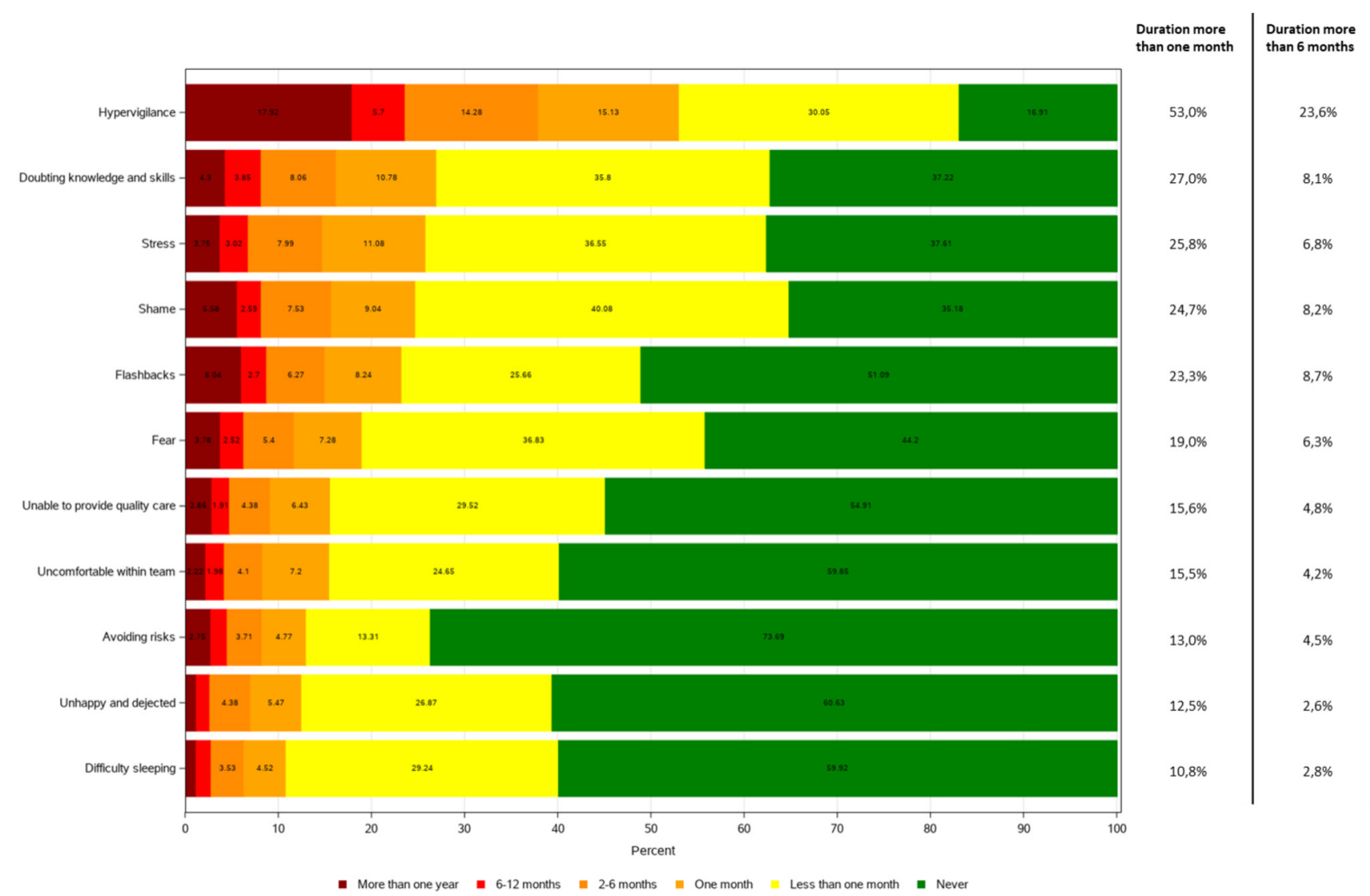

Figure 1 Symptoms in the aftermath of PSI. PSI, patient safety incident.

particularly at risk for poor well-being and reduced professional functioning during those previous 6 months as they were involved in a PSI with such serious sequelae. More than half of all respondents suffered hypervigilance for more than 1 month, while almost one in four suffered more than 6 months. The prevalence ratio for all symptoms increased with a higher degree of patient harm. Interestingly, this was also true for all symptoms; except for feeling unhappy and dejected and difficulty sleeping; 6 months after being involved in a PSI compared with 1 month.

The results reported are in line with previous prevalence reports pertaining to emotional responses. ${ }^{7920} \mathrm{We}$ found that in addition to earlier publications reporting symptoms such as anxiety, flashback and insomnia, that hypervigilance was the most universally reported symptom $(80 \%)$. A recent study of 5782 physician mothers found that involvement in a mistake was associated with higher reported burn-out. ${ }^{20}$ The psychological impact of PSI on the healthcare professional is thought to be similar to that characterising PTSD. ${ }^{21}$ We, therefore, chose a cut-off set at 1 month based on the criteria set by DSM- 5 for PTSD ${ }^{15}$ Suffering symptoms longer than 1 month, let alone 6 months, has a profound impact on healthcare providers' professional and personal life. As personal testimonials reveal, healthcare providers cannot provide the quality of care that is needed and they are at risk of being involved in future PSI's. ${ }^{22}$
Healthcare professionals may, at some point, find themselves in a vicious circle. As healthcare workers involved in PSI are at risk of diminished personal well-being and reduced professional performance adequate institutional and peer support are more and more considered essential components in alleviating the personal and professional impact on these second victims. Healthcare organisations need more awareness that some of the symptoms, such as doubts about knowledge and skill, feeling unable to provide quality care, feeling uncomfortable with the team, may seriously impact team performance, patient safety and the provision of quality care. Organisations should, therefore, play a proactive role in providing immediate support to healthcare professionals involved in a PSI and should not wait until the healthcare professional gets depressed, develops burn-out or quits the job. The risk of not responding to a PSI in a timely and effective manner can have a significant impact on the healthcare professional involved and organisations alike. It can lead to absence of healing, loss of trust, no learning and improvement while it may also increase the likelihood of lawsuits and patient complaints. ${ }^{5}$ Perceptions of second victimness, turnover intentions and absenteeism may be less severe when the healthcare organisation's culture is characterised by a non-punitive response to errors. Such non-punitive response may encourage supportive interactions, openness to discuss error and thus mitigate the negative effects of PSI. ${ }^{21}$ A recent study among Dutch 
Table 3 Overview of symptoms persisting longer than 1 month

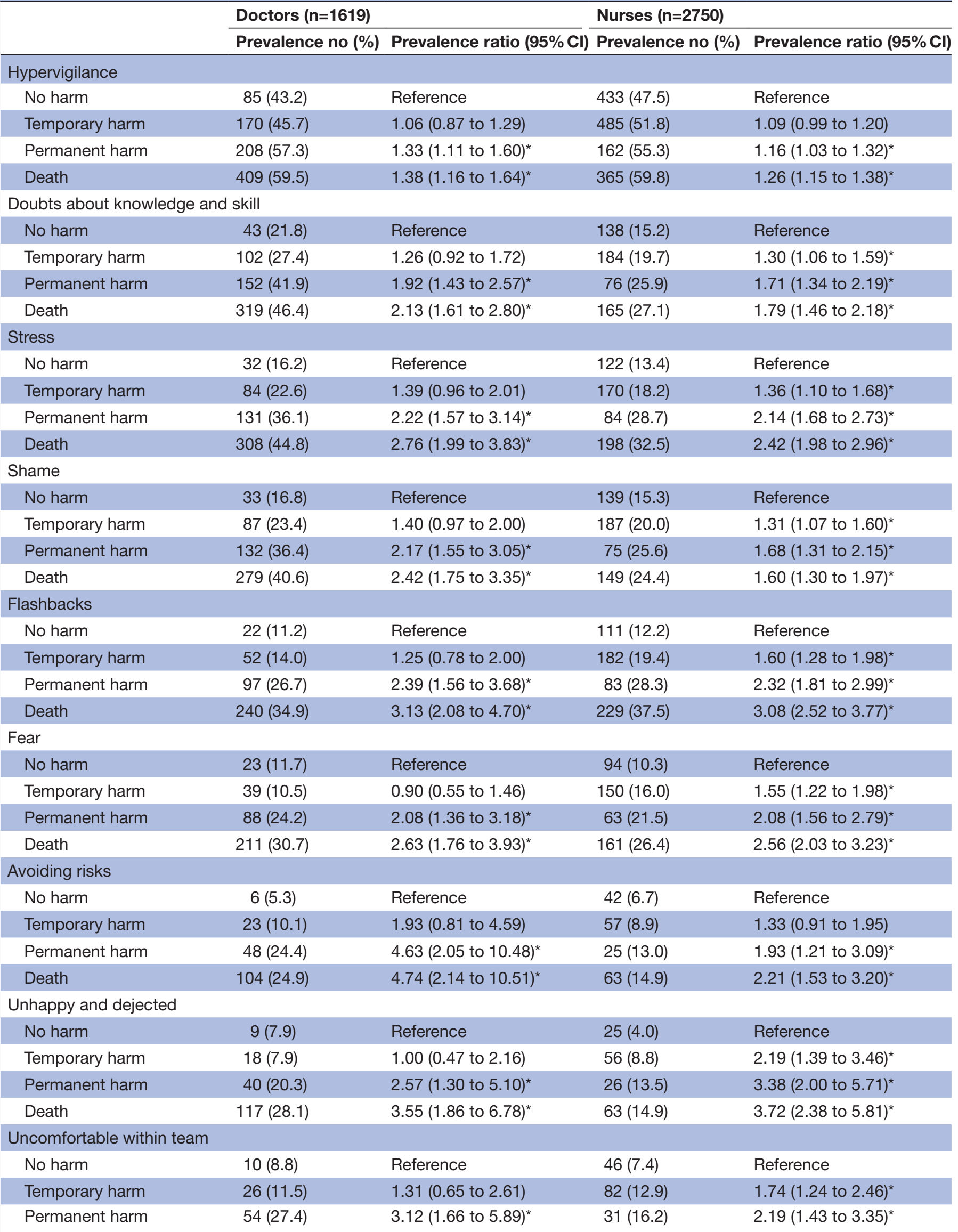


Table 3 Continued

\begin{tabular}{|c|c|c|c|c|}
\hline & \multicolumn{2}{|l|}{ Doctors ( $n=1619)$} & \multicolumn{2}{|l|}{ Nurses $(n=2750)$} \\
\hline & Prevalence no (\%) & Prevalence ratio $(95 \% \mathrm{Cl})$ & Prevalence no (\%) & Prevalence ratio $(95 \% \mathrm{Cl})$ \\
\hline Death & $115(27.6)$ & $3.14(1.70 \text { to } 5.80)^{*}$ & $75(17.7)$ & $2.41(1.70 \text { to } 3.40)^{*}$ \\
\hline \multicolumn{5}{|l|}{ Difficulty sleeping } \\
\hline No harm & $3(2.6)$ & Reference & $23(3.7)$ & Reference \\
\hline Temporary harm & $11(4.9)$ & $1.84(0.52$ to 6.47$)$ & $50(7.8)$ & $2.13(1.31 \text { to } 3.44)^{\star}$ \\
\hline Permanent harm & $31(15.7)$ & $5.98(1.87 \text { to } 19.12)^{\star}$ & $27(14.1)$ & $3.82(2.24 \text { to } 6.50)^{*}$ \\
\hline Death & $94(22.5)$ & $8.57(2.77 \text { to } 26.54)^{\star}$ & $68(16.1)$ & $4.36(2.76 \text { to } 6.88)^{\star}$ \\
\hline \multicolumn{5}{|c|}{ Unable to provide quality care } \\
\hline No harm & $8(7.0)$ & Reference & $41(6.6)$ & Reference \\
\hline Temporary harm & $21(9.3)$ & 1.32 (0.60 to 2.88$)$ & $85(13.3)$ & $2.03(1.42 \text { to } 2.89)^{\star}$ \\
\hline Permanent harm & $46(23.4)$ & $3.33(1.63 \text { to } 6.80)^{*}$ & $39(20.3)$ & $3.09(2.06 \text { to } 4.65)^{\star}$ \\
\hline Death & $106(25.4)$ & $3.62(1.82 \text { to } 7.21)^{*}$ & $95(22.5)$ & $3.42(2.42 \text { to } 4.83)^{\star}$ \\
\hline
\end{tabular}

${ }^{*} \mathrm{P}<0.05$.

gynaecologists showed that $60 \%$ of respondents deemed the current hospital support services after an adverse event insufficient. Two-thirds reported that their department or hospital lacked a support protocol or strategy and they were close to unanimous in preferring support from direct colleagues. ${ }^{23}$

Well-being and joy in work receive important attention nowadays in medical journals. ${ }^{24-28}$ Well-being in physicians and other healthcare professionals has a direct link with patient outcomes. In general, healthcare may need to focus more on positive dimensions like work engagement and enjoy unexpected small positive experiences like Mangomoment a movement recently described by our department and now getting international attention. ${ }^{27}$ The results of this study emphasise that the Triple Aim, a framework, that describes an approach to optimising health system performance is expanded to a Quadruple aim, meaning that next to enhancing patient experience, improving population health and reducing costs, improving the work life of healthcare providers is added to optimise health system performance. ${ }^{29}$ To improve joy in work, it is now time for action by senior leaders, managers and healthcare providers alike.

The large sample size $(\mathrm{n}=6508)$ as well as the large number of Dutch hospitals ( $\mathrm{n}=32$ ) included strengthen this report. However, there are also some limitations. First, even though the participating hospitals vary in size, location and function, the results may not be representative for all hospitals in the Netherlands. Second, the questionnaire was distributed in several ways (by email, by intranet, by chairman of departments) and this implies that no response rate can be calculated. Third, in order to keep the questionnaire concise and little time consuming, we did not make use of extensive validated questionnaires but choose a dichotomous (present or absent) variable to record the symptoms. Last, since we rely on the recollection and perception of the respondents the data may be subject to recollection bias.

Supporting healthcare professionals in the aftermath of a PSI is an important challenge for healthcare professionals, managers, board members and policy-makers. It should be part of the overall quality management system. For example, there should be more awareness about the emotional impact, building a professional support system and improve the notification of the available support mechanisms. This should result in better quality of care to patients and their family as only healthcare professionals who are feeling well and supported by colleagues and boards, are able to provide high quality of care. As the involved patients and family are the main victims of these serious clinical adverse events, all involved clinicians and managers should focus on the needs of these first victims. Open communication on the event and openness about the improvement initiatives should support the safety climate and overall quality of healthcare.

Future studies should focus on how organisations best embed the support of healthcare professionals involved in PSI in their vision, mission and policy regarding patient safety and quality care efforts. Specifically, longitudinal studies focusing on the duration of the symptoms may help in making the current support systems more evidence based. Future work should also include a screening method in order to individualise institutional and peer support in the aftermath of a PSI. More data are needed in order to identify which actions should be taken to reduce the duration of the symptoms for those who suffer longer than expected. We also need to understand more about the degree of well-being of those healthcare providers involved in a PSI resulting in permanent harm or death, reporting no symptoms at all. 
Table 4 Overview of symptoms persisting longer than 6 months

Doctors $(n=1619)$

Prevalence no (\%) Prevalence ratio $(95 \% \mathrm{Cl}) \quad$ Prevalence no (\%)

Hypervigilance

$\begin{array}{lclcl}\text { No harm } & 30(15.2) & \text { Reference } & 206(22.6) & \text { Reference } \\ \text { Temporary harm } & 52(14.0) & 0.92(0.61 \text { to } 1.39) & 206(22.0) & 0.97(0.82 \text { to } 1.15) \\ \text { Permanent harm } & 77(21.2) & 1.39(0.95 \text { to } 2.05) & 88(30.0) & 1.33(1.07 \text { to } 1.64)^{\star} \\ \text { Death } & 182(26.5) & 1.74(1.22 \text { to } 2.47)^{\star} & 191(31.3) & 1.38(1.17 \text { to } 1.64)^{\star}\end{array}$

Doubts about knowledge and skill

\begin{tabular}{|c|c|c|c|c|}
\hline No harm & $5(2.5)$ & Reference & $25(2.7)$ & Reference \\
\hline Temporary harm & $19(5.1)$ & 2.01 (0.76 to 5.31$)$ & $52(5.6)$ & $2.02(1.27 \text { to } 3.23)^{*}$ \\
\hline Permanent harm & 47 (13.0) & 5.10 (2.06 to 12.62$)^{*}$ & $25(8.5)$ & $3.11(1.81 \text { to } 5.33)^{\star}$ \\
\hline Death & $114(16.6)$ & 6.54 (2.71 to 15.78$)^{*}$ & $69(11.3)$ & $4.12(2.64 \text { to } 6.44)^{\star}$ \\
\hline \multicolumn{5}{|l|}{ Stress } \\
\hline No harm & $4(2.0)$ & Reference & $32(3.5)$ & Reference \\
\hline Temporary harm & $15(4.0)$ & 1.99 (0.67 to 5.90$)$ & $49(5.2)$ & 1.49 (0.96 to 2.30$)$ \\
\hline Permanent harm & $29(8.0)$ & $3.93(1.40 \text { to } 11.03)^{\star}$ & $26(8.9)$ & $2.53(1.53 \text { to } 4.17)^{\star}$ \\
\hline Death & 84 (12.2) & $6.02(2.24 \text { to } 16.21)^{*}$ & $57(9.3)$ & $2.66(1.75 \text { to } 4.05)^{*}$ \\
\hline \multicolumn{5}{|l|}{ Shame } \\
\hline No harm & $6(3.1)$ & Reference & $33(3.6)$ & Reference \\
\hline Temporary harm & $21(5.7)$ & 1.85 (0.76 to 4.52$)$ & $67(7.2)$ & $1.98(1.32 \text { to } 2.97)^{\star}$ \\
\hline Permanent harm & $35(9.6)$ & $3.17(1.36 \text { to } 7.40)^{\star}$ & $31(10.6)$ & $2.92(1.82 \text { to } 4.68)^{\star}$ \\
\hline Death & 99 (14.4) & 4.73 (2.11 to 10.62$)^{\star}$ & $65(10.7)$ & $2.94(1.96 \text { to } 4.42)^{*}$ \\
\hline \multicolumn{5}{|l|}{ Flashbacks } \\
\hline No harm & $7(3.6)$ & Reference & $32(3.5)$ & Reference \\
\hline Temporary harm & $17(4.6)$ & 1.29 (0.54 to 3.05$)$ & $68(7.3)$ & $2.07(1.37 \text { to } 3.12)^{\star}$ \\
\hline Permanent harm & $30(8.3)$ & $2.33(1.04 \text { to } 5.20)^{*}$ & $31(10.6)$ & $3.01(1.87 \text { to } 4.85)^{*}$ \\
\hline Death & $94(13.7)$ & $3.85(1.82 \text { to } 8.16)^{*}$ & $103(16.9)$ & $4.81(3.28 \text { to } 7.05)^{\star}$ \\
\hline \multicolumn{5}{|l|}{ Fear } \\
\hline No harm & $3(1.5)$ & Reference & $25(2.7)$ & Reference \\
\hline Temporary harm & $8(2.2)$ & 1.41 (0.38 to 5.26$)$ & $48(5.1)$ & $1.87(1.16 \text { to } 3.00)^{*}$ \\
\hline Permanent harm & $26(7.2)$ & $4.70(1.44 \text { to } 15.34)^{*}$ & $24(8.2)$ & $2.98(1.73 \text { to } 5.14)^{*}$ \\
\hline Death & $79(11.5)$ & 7.55 (2.41 to 23.66$)^{*}$ & $62(10.2)$ & $3.70(2.35 \text { to } 5.83)^{*}$ \\
\hline \multicolumn{5}{|l|}{ Avoiding risks } \\
\hline No harm & $1(0.9)$ & Reference & $13(2.1)$ & Reference \\
\hline Temporary harm & $8(3.5)$ & $4.02(0.51$ to 31.73$)$ & $25(3.9)$ & 1.88 (0.97 to 3.64$)$ \\
\hline Permanent harm & $13(6.6)$ & 7.52 (0.99 to 56.76$)$ & $8(4.2)$ & 2.00 (0.84 to 4.75$)$ \\
\hline Death & $33(7.9)$ & $9.02(1.25 \text { to } 65.25)^{*}$ & $27(6.4)$ & $3.06(1.60 \text { to } 5.87)^{*}$ \\
\hline \multicolumn{5}{|c|}{ Unhappy and dejected } \\
\hline No harm & $2(1.8)$ & Reference & $3(0.5)$ & Reference \\
\hline Temporary harm & $2(0.9)$ & 0.50 (0.07 to 3.52$)$ & $11(1.7)$ & $3.59(1.01 \text { to } 12.79)^{\star}$ \\
\hline Permanent harm & $7(3.6)$ & $2.03(0.43$ to 9.59$)$ & $5(2.6)$ & $5.42(1.31 \text { to } 22.46)^{*}$ \\
\hline Death & $30(7.2)$ & $4.10(0.99$ to 16.90$)$ & $15(3.6)$ & $7.38(2.15 \text { to } 25.32)^{\star}$ \\
\hline \multicolumn{5}{|c|}{ Uncomfortable within team } \\
\hline No harm & $1(0.9)$ & Reference & $8(1.3)$ & Reference \\
\hline Temporary harm & $7(3.1)$ & 3.52 (0.44 to 28.23$)$ & $21(3.3)$ & $2.57(1.15 \text { to } 5.75)^{\star}$ \\
\hline Permanent harm & $10(5.1)$ & 5.79 (0.75 to 44.62$)$ & $10(5.2)$ & $4.06(1.63 \text { to } 10.15)^{\star}$ \\
\hline
\end{tabular}

Continued 
Table 4 Continued

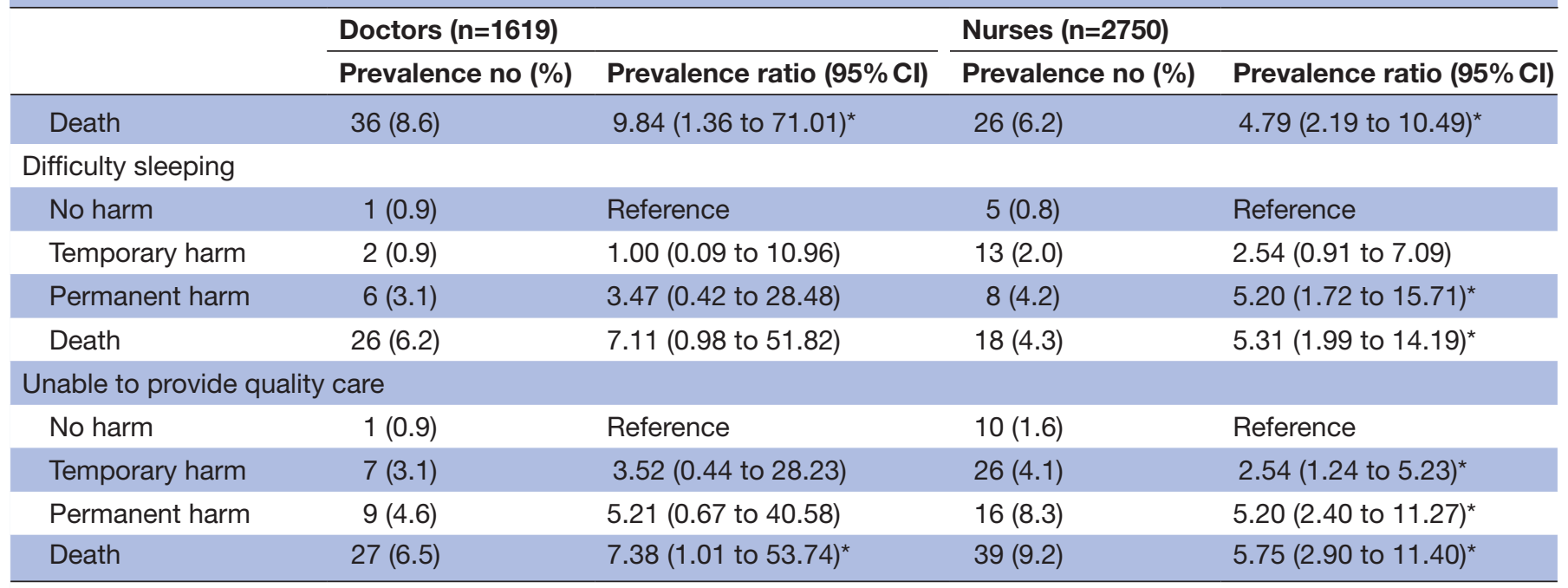

${ }^{*} \mathrm{P}<0.05$

\section{CONCLUSION}

In conclusion, the majority of doctors and nurses in approximately one-third of Dutch hospitals involved in a PSI at least once during their career and who chose to participate in this study report having suffered several symptoms in the aftermath of the PSI. Some of these symptoms lasted longer than 1 month while others, for example, hypervigilance, even lasted longer than 6 months. Attention should be given to how to cope with these symptoms as they profoundly affect personal wellbeing, professional performance as well as teamwork-related efforts directly influencing patient safety and the provision of quality care.

\section{Author affiliations}

${ }^{1}$ Leuven Institute for Healthcare Policy - Department of Public Health and Primary Care, KU Leuven - University of Leuven, Leuven, Belgium

${ }^{2}$ Department of Quality Management, University Hospitals Leuven, Leuven, Belgium ${ }^{3}$ Management Consultant \& coordinator Peer Support Learning Network, Utrecht, The Netherlands

${ }^{4}$ Department of Quality Management, University Hospitals Leuven, Leuven, Belgium ${ }^{5}$ Department of Translational Medicine, University of Eastern Piedmont - UPO, Novara, Italy

${ }^{6}$ Tjongerschans Hospital, Heerenveen, The Netherlands

Collaborators Member of the Dutch Peer Support Collaborative Research Group are: Mulders IAH, Hopstaken LEM, del Canho R, Lim C, Diekerhof C, Hoppenbrouwers SJM, Doreleijers CTJM, Groenhuijzen A, den Dijker L, Simons PJ, Cornelissen NMP, Derwig JJC, Wennekes MJ, van den Driessche M, Lim FTH, So RKL, Blaauw J, ten Brummelhuis I, Withaar R, van Schie M-J, Alders AM, Möllers MJW, Schuurman G, Maas-Engelen S, ten Have SMTH, Rhodius RJ, Fakkedij TMV, van Hasselt DG, Roosen AEM, Vanhaecht Kris, Department of Quality Management, University Hospitals Leuven, Belgium \& Leuven Institute for Healthcare Policy - Department of Public Health and Primary Care, KU Leuven - University of Leuven, Leuven, Belgium. Seys Deborah, Leuven Institute for Healthcare Policy Department of Public Health and Primary Care, KU Leuven - University of Leuven, Leuven, Belgium. Schouten Loes, Management Consultant \& coordinator Peer Support Learning Network, Utrecht, The Netherlands. Bruyneel Luk, Department of Quality Management, University Hospitals Leuven, Belgium \& Leuven Institute for Healthcare Policy - Department of Public Health and Primary Care, KU Leuven - University of Leuven, Leuven, Belgium Coeckelberghs Ellen, Leuven Institute for Healthcare Policy - Department of Public Health and Primary Care, KU Leuven
- University of Leuven, Leuven, Belgium Panella Massimiliano, Department of Translational Medicine, University of Eastern Piedmont - UPO, Novara, Italy Zeeman G. Gerda, Department of Obstetrics \& Gynaecology, Tjongerschans Hospital; Heerenveen, The Netherlands.

Contributors Conception and design of the study: KV, LS, MP and GZ. Acquisition of the data: LS, EC and GZ. Analysis and interpretation of the data: KV, DS, LB and EC. Drafting of the manuscript: DS, LS, LB, GZ and KV. All authors critically revised the manuscript for intellectual content and approved the final version.

Funding This study was carried out with an unconditional grant from VvAA. Competing interests None declared.

Patient consent for publication Not required.

Provenance and peer review Not commissioned; externally peer reviewed.

Data sharing statement All data relevant to the study are included in the article or uploaded as online supplementary information.

Open access This is an open access article distributed in accordance with the Creative Commons Attribution Non Commercial (CC BY-NC 4.0) license, which permits others to distribute, remix, adapt, build upon this work non-commercially, and license their derivative works on different terms, provided the original work is properly cited, appropriate credit is given, any changes made indicated, and the use is non-commercial. See: http://creativecommons.org/licenses/by-nc/4.0/.

\section{REFERENCES}

1. Classen DC, Resar R, Griffin F, et al. 'Global trigger tool' shows that adverse events in hospitals may be ten times greater than previously measured. Health Aff 2011;30:581-9.

2. de Vries EN, Ramrattan MA, Smorenburg SM, et al. The incidence and nature of in-hospital adverse events: a systematic review. Qual Saf Health Care 2008;17:216-23.

3. Wu AW, Aw W. Medical error: the second victim. The doctor who makes the mistake needs help too. BMJ 2000;320:726-7.

4. Clarkson MD, Haskell $\mathrm{H}$, Hemmelgarn $\mathrm{C}$, et al. Abandon the term "second victim". BMJ 2019;364:I1233.

5. Conway J, Federico F, Stewart K, et al. Respectful management of serious clinical adverse events. IHI Innovation Series white paper. Cambridge, Massachusetts: Institute for Healthcare Improvement, 2011. Available on. http://www.ihi.org/

6. Harrison R, Lawton R, Stewart K. Doctors' experiences of adverse events in secondary care: the professional and personal impact. Clin Med 2014:14:585-90.

7. Mira JJ, Carrillo I, Lorenzo S, et al. The aftermath of adverse events in Spanish primary care and hospital health professionals. BMC Health Serv Res 2015;15:151. 
8. Coughlan B, Powell D, Higgins MF. The Second Victim: a Review. Eur J Obstet Gynecol Reprod Biol 2017;213:11-16.

9. Seys D, Wu AW, Van Gerven E, et al. Health care professionals as second victims after adverse events: a systematic review. Eval Health Prof 2013;36:135-62.

10. Van Gerven E, Bruyneel L, Panella M, et al. Psychological impact and recovery after involvement in a patient safety incident: a repeated measures analysis. BMJ Open 2016;6:e011403.

11. Han K, Bohnen JD, Peponis T, et al. The Surgeon as the Second Victim? Results of the Boston Intraoperative Adverse Events Surgeons' Attitude (BISA) Study. J Am Coll Surg 2017;224:1048-56.

12. Chan ST, Khong PCB, Wang W. Psychological responses, coping and supporting needs of healthcare professionals as second victims. Int Nurs Rev 2017;64:242-62.

13. Van Gerven E, Vander Elst T, Vandenbroeck S, et al. Increased Risk of Burnout for Physicians and Nurses Involved in a Patient Safety Incident. Med Care 2016;54:937-43.

14. Robertson N, Perry A. Institutionally based health care workers exposure to traumatogenic events: systematic review of PTSD presentation. J Trauma Stress 2010;23:417-20.

15. American Psychiatric Association. Diagnostic and Statistical Manual of Mental Disorders (DSM-5). https://www.psychiatry. org/psychiatrists/practice/dsm?_ga=1.8367346.1782582538. 1481136819.

16. Tamhane AR, Westfall AO, Burkholder GA, et al. Prevalence odds ratio versus prevalence ratio: choice comes with consequences. Stat Med 2016;35:5730-5.

17. Van Gerven E, Deweer D, Scott SD, et al. Personal, situational and organizational aspects that influence the impact of patient safety incidents: A qualitative study. Rev Calid Asist 2016;31 Suppl 2(Suppl 2):34-46.
18. Van Gerven E, Seys D, Panella M, et al. Involvement of healthcare professionals in an adverse event: the role of management in supporting their workforce. Pol Arch Med Wewn 2014;124:312-20.

19. Van Gerven E, Vanhaecht K, Euwema M, et al. Health professionals as second victims of patient safety incidents: impact on functioning and well-being, 2016.

20. Gupta K, Lisker S, Rivadeneira NA, et al. Decisions and repercussions of second victim experiences for mothers in medicine (SAVE DR MoM). BMJ Qual Saf 2019;28:564-73.

21. Quillivan RR, Burlison JD, Browne EK, et al. Patient safety culture and the second victim phenomenon: Connecting culture to staff distress in nurses. Jt Comm J Qual Patient Saf 2016;42:377-86.

22. Buikema M. When healthcare hurts: doctors share their darkest hours: Zin Publishing, 2011.

23. Baas MAM, Scheepstra KWF, Stramrood CAl, et al. Work-related adverse events leaving their mark: a cross-sectional study among Dutch gynecologists. BMC Psychiatry 2018;18:73.

24. Thomas LR, Ripp JA, West CP. Charter on physician well-being JAMA 2018;319:1541-2.

25. Schwenk TL. Physician well-being and the regenerative power of caring. JAMA 2018;319:1543-4.

26. Perlo J, Balik B, Swensen S, et al. IHI Framework for Improving Joy in Work. IHI White Paper. Cambridge, Massachusetts: Institute for Healthcare Improvement, 2017.

27. Vanhaecht K. In search of Mangomoments. Lancet Oncol 2018;19:165.

28. Perlo J, Feeley D. Why focusing on professional burnout is not enough. J Healthc Manag 2018;63:85-9.

29. Bodenheimer T, Sinsky C. From triple to quadruple aim: Care of the patient requires care of the provider. Ann Fam Med 2014;12:573-6. 